\begin{tabular}{|l|l|l|}
\hline \multicolumn{2}{|c|}{ PublisherInfo } \\
\hline \hline PublisherName & $:$ & BioMed Central \\
\hline \hline PublisherLocation & $:$ & London \\
\hline \hline PublisherImprintName & $:$ & BioMed Central \\
\hline \hline
\end{tabular}

\title{
Nuclear cytometric changes in breast carcinogenesis
}

\begin{tabular}{|l|l|l||}
\hline \multicolumn{2}{|c||}{ ArticleInfo } \\
\hline \hline ArticleID & $:$ & 3753 \\
\hline \hline ArticleDOI & $:$ & $10.1186 /$ bcr-2000-66716 \\
\hline \hline ArticleCitationID & $:$ & 66716 \\
\hline \hline ArticleSequenceNumber & $:$ & 25 \\
\hline \hline ArticleCategory & $:$ & Paper Report \\
\hline \hline ArticleFirstPage & $:$ & 1 \\
\hline \hline ArticleLastPage & $:$ & 4 \\
\hline \hline & $:$ & RegistrationDate : 2000-10-31 \\
ArticleHistory & $:$ & OnlineDate \\
\hline \hline ArticleCopyright & $:$ & Current Science Ltd2000-10-31 \\
\hline \hline ArticleGrants & $:$ & \\
\hline \hline ArticleContext & $:$ & 1305833 \\
\hline \hline
\end{tabular}


Aff1 Baylor College of Medicine, Houston, Texas, USA

\section{Keywords}

Atypical, hyperplasia, image analysis, in situ carcinoma, premalignant breast lesions

\section{Introduction}

Image morphometry has been shown to provide useful information in several types of human tumors, including breast. Image cytometry can identify preinvasive lesions in human cervix with malignant potential and may predict response in patients with prostate and renal carcinoma. In breast cancer, it has been shown that morphometric data correlates with several important biological factors, including hormone receptors and p53 status. However, premalignant mammary lesions have not been studied in a systematic fashion using this technique.

\section{Aims}

To study quantitated nuclear morphological features of the entire spectrum of human premalignant breast lesions including UDH, atypical ductal hyperplasia (ADH), DCIS and IC.

\section{Comments}

This study evaluated the entire spectrum of human premalignant breast lesions by detailed image morphometry. Overall the findings corroborate similar data obtained by light microscopy and molecular genetics. Based on their observations, the authors have suggested a model of progression from well differentiated ductal carcinoma in situ (DCIS) to well differentiated invasive carcinoma (IC) and poorly differentiated DCIS to poorly differentiated IC. The major weakness of this study is that the presence of such a model is difficult to ascertain if 'intermediate' differentiated lesions are not studied. Indeed, most experts in this field believe that DCISs are so heterogeneous that it is extremely difficult to meaningfully dichotomize them as low or high grade. ICs have traditionally been classified into three or sometimes four grades. The results of this study suggest that morphometric features may help segregate 'intermediate' differentiated DCIS and IC into clinically useful subgroups. It has been shown that genetic 
alterations can be detected in 'normal' epithelium obtained from breasts harboring malignancy. This suggests that morphologic changes lag behind genetic alterations. It appears from the figures in the paper that there are subtle but still recognizable differences between 'normal' epithelium in terminal duct lobular units (from reduction mammoplasties) and mild/moderate usual ductal hyperplasia (UDH). It would be interesting to use this technique to study 'normal' epithelium adjacent to cancer to find morphometric features that identify cells that may harbor genetic alterations.

\section{Methods}

Modified Fuelgen staining was performed on $71 \mathrm{UDH}, 9$ ADH, 24 DCIS and 43 IC. Intermediately differentiated DCIS and IC were excluded. About 50-300 non-overlapping nuclei were imaged on a Cyto-Savant imaging device. Nuclear features $(n=128)$ were analyzed in the following categories; morphometric, photometric, discrete texture, Markovian texture, fractal texture and run length texture features. Normal lymphocytes were used as internal diploid controls.

\section{Results}

Nuclei in more advanced lesions generally displayed the following features: they were larger; were more pleomorphic; showed a reduced area with high density chromatin; showed decreased contrast; showed increased fractal area; and showed increased mean value of optical intensity. The lesions could be divided into three groups with similar features: (1) normal epithelium (from reduction mammoplasties), mild and moderate UDH; (2) florid UDH, ADH, well differentiated DCIS and well differentiated IC, and (3) poorly differentiated DCIS and high grade IC. DNA index showed that florid $\mathrm{UDH}$ is polyclonal and some of these lesions contain aneuploid clones. Of the 128 nuclear features studied, 56 were significantly different between well and poorly differentiated DCIS and IC.

\section{Discussion}

This study suggests that morphometric characteristics of epithelial cells in the terminal duct lobular unit are similar to those in mild and moderate UDH. Some florid UDH contain aneuploid cell population and may represent biologically more advanced lesions. Similar morphometric features of ADH and well differentiated DCIS suggest that these two lesions are nearly equally advanced and may differ is size only. Features of ADH, well differentiated DCIS and IC are remarkably different from poorly differentiated DCIS and IC and support the hypothesis of direct biologic progression in these categories. 


\section{References}

1. Mommers ECM, Poulin N, Sangulin J, Meijer CJLM, Baak JPA, van Diest PJ: Nuclear cytometric changes in breast carcinogenesis. J Pathol. 2000, In Press: 4592-4603.

This PDF file was created after publication. 